\title{
LA CRÍTICA EN LA ENSEÑANZA DE LAS CIENCIAS: CONSTRUCTIVISMO Y CONTRADICCIÓN
}

LABURU, C.E.

Departamento de Física.Universidad de Londrina, Brasil.

\section{SUMMARY}

In this study we try to justify the importance of considering in science teaching that facet of critical thinking that is based on the idea of comparing the evidence with the prevision. Together with critical thinking, the most general and fundamental condition of coherent thinking must prevail. This viewpoint is justified by employing the popperian epistemological model, the cognitive model by Piaget and the model of conceptual change. We show that for these models, contradiction is an essential epistemological element. We suggest that critical thinking should be the principal object of teachers in the science class.

«La claridad y la distinción no constituyen criterios de verdad, pero la oscuridad y la confusión pueden indicar el error. De la misma forma, la coherencia, no puede por sí misma establecer la verdad, pero la incoherencia y la inconsistencia revelan la falsedad.»

Popper

\section{INTRODUCCIÓN}

De entre las muchas cuestiones que se pueden plantear a los educadores en general y, en particular, a aquéllos que se interesan por la enseñanza de ciencias, el problema de establecer el objetivo de la educación científica debería ser una preocupación siempre presente. $O$, más directamente, ¿qué se espera como resultado de una actividad educadora en ciencias? O también ¿qué se espera que obtengan los estudiantes a través de la educación científica?

Nuestra convicción de que las teorías pedagógicas se aproximan más a saberes ligados a la coordinación de valores y, por tanto, sin pretender alcanzar el estatus de ciencia -como aftrma Piaget (1983) para el caso de la filosofía - hace que orientemos las respuestas a las preguntas anteriores siguiendo un criterio de valor. Ese criterioes el que establece acciones pedagógicas que den importancia a la enseñanza crítica.

Por otro lado, una posible respuesta directa que tenga en consideración el criterio anterior sin duda estatía orientada a la adquisición del conocimiento de contenidos científicos ya establecidos socialmente.
Este trabajo propone que, durante el proceso de aprendizaje de contenidos, el papel del educador en ciencias se distinga no sólo por el control de la construcción de conocimientos específicos sino, también, por la responsabilidad de desarrollar simultáneamente la actitud crítica de quienes aprenden. Con eso creemos que el profesor, al proceder de esta forma, está ayudando a sus alumnos al desarrollo de una actitud crítica y mejorando, aś́, la construcción del contenido deseado.

A lo largo de esta discusión es nuestra intención mostrar que el control de la construcción del conocimiento de los alumnos, desde la perspectiva constructivista, es inseparable de la enseñanza crítica.

Partiendo del objetivo educativo de promover el pensamiento crítico, el trabajo intentará indicar implícitamente que la actitud crítica responde a otra cuestión más general: ¿cómo se pasa de un estado de menor conocimiento a uno de mayor?

Esta pregunta nos llevará a desarrollar brevemente dos perspectivas, una epistemológica y otra psicológico- 
cognitiva, ambas centradas en la crítica como un elemento responsable del aumento de conocimientos. La primera perspectiva se basará en las ideas popperianas sobre la naturaleza del conocimiento científico y su desarrollo. La segunda tendrá como soporte la teoría de equilibración cognitiva de Piaget.

Tras la presentación de esas dos perspectivas detallaremos el modelo de enseñanza-aprendizaje constructivista, consolidado desde los años ochenta hasta nuestros días, tanto en lo que se refiere a la investigación en la enserianza de las ciencias como en sus consecuencias en la práctica pedagógica. Mostraremos que el pensamiento crítico es un elemento central dentro de la concepción constructivista.

Por tanto, el principal interés de este trabajo se refiere al conocimiento científico, y está dirigido al profesor de ciencias, particularmente a los de enseñanza secundaria y universitaria.

Al centrar la discusión del pensamiento crítico en los tres campos mencionados del saber -el epistemológico, que intenta comprender la naturaleza y el progreso del conocimiento cientifico construido filogenéticamente; el psicológico-cognitivo, que se caracteriza por la formacion y aumento del conocimiento ontogenético; y el pedagógico, que procura culminar el proceso de enseñanzaaprendizaje en un conocimiento de contenido específico-, queremos demostrar que la crítica estâ considerada como un denominador conceptual común a esos campos de conocimiento.

Aclaremos, antes de presentar las tres secciones propuestas, que al hablar de pensamiento crítico estamos indicando también aquellos pensamientos que exploran la contradicción. La concepción de contradicción debe ser entendida tanto en el plano conceptual, a través de hacer explícitas las incoherencias, como en el plano empírico (especialmente tratándose de la enseñanza de ciencias) donde la negación mediante evidencias comprueba la insuficiencia de determinadas inferencias.

Al imaginar un pensador critico, se debe considerar un pensador comprometido a la vez con la coherencia interna y con la generalidad.

Además de ser importante para una actitud pedagógica implicada en la superación de aquellas incoherencias que resultan invalidadas por evidencias contrarias a las inferencias previas (previsión teórica, test empírico, nueva prevision teorica para compatibilizar el refutador empírico), hay que reconocer que un pensamiento científico-crítico exige otras actitudes igualmente relevantes: necesidad de experimentar, observar, argumentar, crear y estar inclinado a enjuiciar, procurando fundamentar estos juicios, así como dar razones que demanden justificaciones, estando capacitado para investigar y explorar afirmaciones no tratadas. Gil (1993), por ejemplo, insiste en que un cambio conceptual está básicamente vinculado a un cambio metodológico y afirma que el elevado fracaso en la resolución de problemas -(de Iápiz y papel, Gil y Dumas-Carré, 1990)- se debe a la metodología del sentido común empleada por los alumnos en esa resolución (metodología de la superficialidad, Gil y Carrascosa 1985). Por otro lado, ese fracaso se reduce cuando los estudiantes aprenden Ia metodología de la investigación científica. Para Siegel (1989), las razones, las justificaciones y el acto de inquerir caracterizan el espíritu crítico. De la misma forma, Désautels y Larochelle (1990) condicionan el pensamiento crítico a la posibilidad de establecer las bases de nuestras ideas, en la medida en que se den las razones de los porques. Añaden que un pensamiento crítico está basado en un razonamiento autocrítico y flexible, en oposicion a un razonamiento rígido que examine nuestras creencias y fundamentos, llevando a una reflexión sobre las propias reflexiones.

Sin despreciar la relevancia de los conceptos anteriores, subrayamos que este trabajo pretende centrarse en la crítica que resulta de la evidencia de las contradicciones, con el fin de confrontar las ideas de quienes aprenden. El profesor de ciencias se compromete con la enseñanza crf́tica cuando procura establecer un clima en la clase en el cual la construcción del conocimiento científico se realiza mediante el empleo explícito de contradicciones como mecanismo de superación de concepciones y al requerir simultáneamente de los estudiantes el constante mantenimiento de comportamientos que impliquen la coherencia y la generalización de sus afirmaciones (Hewson, 1985). Por tanto, podemos afirmar que, al proceder de esa forma, el profesor está coordinando acciones pedagógicas en ciencias directamente ligadas a la línea de enseñanza-aprendizaje constructivista.

Como último comentario es preciso decir que en la propuesta aquí presentada subyace una idea central de racionalidad: la racionalidad, en el caso de la ciencia, es, segtin Siegel, consecuencia directa de su método (método cientffico), que consiste en comprometerse con la evidencia (Siegel, 1989). Para él, la ciencia es racional en la medida en que sus procedimientos, teorías, hipote. sis y afirmaciones son evaluadas apelando a las eviden. cias relevantes.

Aparte de la valoración de este particular criterio de racionalidad científica como objetivo educativo, es preciso dejar claro que somos conscientes de que propuestas como las de Kuhn y Feyerabend indican que este criterio es producto de una cultura cuya racionalidad ha dado mucha importancia a la ciencia (Laudan, 1977). Por consiguiente, s6lo estamos des. tacando una posicion que creemos dominante en el pensamiento cientifico.

Como discutiremos en las secciones siguientes, a pesar de la importancia que se quiere dar a la contradicción, existen aún varias formas de poder evitarla. Franco y Colinvaux-de-Dominguez (1992) llegan a afirmar que la coherencia interna de un sistema es un aspecto distintivo de la ciencia consolidada, y no del proceso de hacer ciencia que siempre alcanza esa coherencia por postulación ad hoc. 


\section{LA CRÍTICA EN LA EPISTEMOLOGÍA}

Dentro de las pretensiones especificadas anteriormente destaquemos, en la epistemología, la teoría del conocimiento popperiana. Popper, fundamentalmente, se preocupa de establecer una demarcación entre el conocimiento científico, por una parte, y el pseudocientífico o sistemas metafísicos, por otra. Afirma que la base del progreso del conocimiento cientifico es la constante búsqueda de la crítica. Un conocimiento se dice científico cuando puede ser sometido a crítica, puesto a prueba, refutado. Debe ser entendida como el enfrentamiento entre teoría y realidad, de donde surgen contradicciones; es decir, nuevos problemas. En otras palabras, hecha una previsión -a la luz de una conjetura teórica-y en caso de que los hechos se resistan (lo que ocurre frecuentemente), aparece un problema. La solución del nuevo problema se da sobre nuevas bases teóricas inventadas o creadas para éste (Popper 1972), «el conocimiento es una aventura con ideas" (Popper 1972).

En la concepción popperiana, la contradicción se origina a partir de la crítica constante, a través de la falsación -mediante pruebas rigurosas- de una conjetura (teoría) respecto a los hechos. Así, Ia búsqueda por la superación de la crítica - de la contradicción-conduce al aumento del conocimiento (Popper 1987).

Para Popper (1972), «criticar consiste invariablemente en resaltar contradicciones [...] en un sentido muy importante, la crítica es el motor principal de cualquier progreso intelectual [...] sin contradiccion y sin crítica, no habría motivos racionales para alterar nuestras teorías -en consecuencia dejaría de haber progreso intelectual (p. 346) [...] es exclusivamente decisión nuestra no admitir la contradicción que nos hace buscar otro punto de vista, lo que nos permite evitaria (p. 348) [...] Así, una teoría que implica una contradicción es enteramente inúti como teoría (p. 350) [...] si buscamos una teoría mejor, to hacemos por creer que la teoría que describimos es mala, debido a las contradicciones que presenta. La aceptación de contradicciones lleva al fin de la crítica $y$, por tanto, al colapso de la ciencia (p. 352) y al fin: aprendemos con las refutaciones, a través de la eliminación de errores, por retroacción» (Popper, 1987).

Aigunos filósofos de la ciencia scríalan la exageración popperiana respecto al criterio falsacionista. Lakatos (1979), por ejemplo, señala que es normal que ias teorías sobrevivan o convivan constantemente con refutaciones. Laudan (1977), por otro lado, advierte que determinados problemas' solamente son considerados así cuando están incluidos en determinados contextos, y que Io que puede ser un problema para determinadas teorías no lo será para otras. El programa epistemológico de Laudan también afirma que es inherente al proceso científico la convivencia con anomalías y problemas conceptuales (Laudan, 1977).

Las inconsistencias en el proceso de comprensión -epistemologico- condicionan la búsqueda de nuevos caminos explicativos, de nuevas ideas; sin embargo, las limitaciones de la epistemología popperiana en su inter- pretación del desarrollo de la ciencia reciben más críticas. La idea central de esta epistemología es su mayor compromiso con la superación de las inconsistencias, encarando las anomalías y los problemas conceptuales como desafíos necesarios para hacer coherente el sistema conceptual. La existencia de incoherencias dentro de dicho sistema es un factor de ruptura potencial del conocimiento establecido y, al restablecer la armonía, impulsaremos el avance científico.

Para las epistemologías anteriormente citadas, incluyendo la de Kuhn (1987), Ia función más importante de la ciencia es la resolución de problemas y éstos pueden, muchas veces, ser considerados como contradicciones que deberán ser solucionadas (nota 3 de la página 18 ).

\section{LA CRÍTICA EN LA PSICOLOGÍA COGNITIVA}

La teoría cognitiva que discutiremos en esta sección está basada en la teoría de la equilibración piagetiana. Pretendemos evidenciar el eje funcionalista de la teoría en detrimento de su eje estructuralista (que realza los estadios) más usual, como hacen algunos trabajos (Rowell, 1983a, 1989; Dykstra, 1992).

La teoría de la equilibración piagetiana pretende entender la formación del conocimiento, desde sus orígenes en la niñez hasta la adolescencia, donde aparecen los orígenes del conocimiento científico. De ella podemos concluir que la evolución del conocimiento individual está caracterizado por la búsqueda de la coherencia (Piaget, 1977; Piaget en: Inhelder, García y Vonèche, 1978).

Para Piaget el sistema cognitivo actúa sobre lo real, a fin de satisfacer necesidades -se considera implícito el carácter motivacional de este proceder (Piaget, 1977). Esta acción (que comprende no sólo procedimientos prácticos, sino también acciones de pensamiento) se ejecuta partiendo de esquemas previos ya construidos por el sujeto.

La esencia de esta teoría es visualizar el sistema cognitivo como un mecanismo formado por dos componentes indisociables: asimilación-acomodación. Este binomio representa dos polos de una interacción que se desarrolla, constituyendo la condición indispensable de todo funcionamiento intelectual. La asimilacion se caracteriza por su naturaleza conservadora y tiende a someter el medio a sus estructuras o esquemas previamente organizados, mientras que la acomodación es fuente de transformaciones y sujeta el sistema cognitivo a las sucesivas imposiciones del medio (Piaget, 1970). Asimilación y acomodación predominan alternativamente, pero siempre tendiendo al equilibrio, dependiendo mutuamente.

El sujeto, interactuando con el objeto, intenta asimilarlo a sus esquemas. Cuando el objeto presente novedades, 
resistiendo a las acciones del sujeto, aparece una situación de desequilibrio de las estructuras lógico-conceptuales del sujeto. Piaget afirma que, para que se restablezca un nuevo equilibrio (más completo en relación con el anterior, Piaget, 1977), debe haber un esfuerzo asimilador (Castro, 1974), siendo necesarias adaptaciones que asimilen nuevamente las resistencias del objeto. En términos más directos, al tratar de satisfacer una necesidad frente a un objeto (real o intelectual), el sujeto parte de estructuras previamente construidas. Esto se traduce en expectativas que se pueden frustrar por una reacción inesperada del objeto -causada por la insuficiencia de estas estructuras-, surgiendo un problema potencial. Así, las estructuras anteriores deben sufrir una transformación, que las haga jerárquicamente superiores, para que la nueva reacción deje de ser inesperada, pasando a formar parte de las expectativas (de los esquemas asimiladores) y de las previsiones del sujeto.

Sin embargo, está claro que toda acomodación siempre parte de esquemas ya construidos y no del vacío. Para acomodar es necesario una base de esquemas de asimilación.

Al existir una previsión insatisfecha, se genera un estado de conflicto (Piaget, 1977) en el sujeto, caracterizado por una situación desequilibradora, o sea, por una contradicción (Piaget, 1977) que para Piaget es el motor de la investigación of fuente real de progreso (Piaget, 1977). Solamente ocurre tal progreso al ser superada la contradicción, después de una nueva reequilibración.

La teoría de Piaget prevé que en función de una perturbación externa, que explicita una contradicción, el mecanismo de equilibración (asimilación-acomodación) ocurra a través de tres fases o comportamientos compensatorios que se oponen a la perturbación:

- Un comportamiento alfa, en el que prevalece el intento de neutralizar la perturbación: considerándola anómala, sin atribuirle importancia o deformándola para no reconocerla como perturbación. Esta manera de restaurar el equilibrio sólo es parcialmente compensadora y el equilibrio es frágil e inestable, fácilmente perturbable.

- Un comportamiento beta, que busca integrar la perturbación en el sistema, sin ignorarla pero creando teorías específicas para explicarla. El sistema comienza a ser modificado hasta alcanzar un nuevo equilibrio en el cual las perturbaciones aparecen como variaciones de la propia estructura reorganizada.

- La reorganización iniciada en beta es completada en el comportamiento gamma que consiste en anticipar por previsión o deducción las posibles variaciones. Se eliminà así la perturbación como tal, incluyéndola en el sistema ya transformado para mantenerla como una posibilidad más y no como una perturbación (Piaget, 1977).

Como ilustración de las ideas anteriores, citemos el siguiente ejemplo (Carvalho et al., 1993): veamos cómo un alumno se puede comportar cuando mide la temperatura de ebullición del agua en una ciudad situada por encima del nivel del mar. Puede esperar, de acuerdio con informaciones anteriores, que el agua hervirá a $1000^{\circ} \mathrm{C}$. $\mathrm{Al}$ obtener experimentalmente un valor menor que éste, presentará un comportamiento alfa cuando se niegue a reconocer dicha perturbación, atribuyendo la anomalía a un defecto del termómetro, o a la incapacidad de la fuente de calor para elevar más la temperatura «si usamos un fuego más alto la temperatura llegará a $\left.100^{\circ} \mathrm{C} »\right)$, o al hecho de que el día sea frío.

Este comportamiento evolucionará hacia una fase beta cuando el alumno intente modificar su explicación, teniendo en cuenta el hecho perturbador. Puede atribuir la menor temperatura al hecho de que el vapor «está transportando el calor's, impidiendo que la tempcratura se eleve, o concluir que la altitud influye en la temperatura de ebullición. Esta evolución culminará en la fáse gamma cuando el alumno posea articuladamente todas la informaciones necesarias para considerar la perturbación como algo previsible dentro de su sistema cognitivo. Para presentar un comportamiento gamma el alumno deberá ser capaz de articular varios esquemas y saber aplicarlos al fenómeno en cuestión. En el ejemplo citado, ello implica coordinar las siguientes cuestiones: un líquido entra en ebullición cuando su presión de vapor iguala a la presión atmosférica; la temperatura a la que esto ocurre es tanto menor cuanto más baja sea la presión atmosférica; la presión atmosférica es menor en altitudes mayores.

La asimilación de toda perturbación externa implica un proceso de acomodación que se inicia en la compensación alfa y se completa en la compensación gamma. Este comportamiento del sistema cognitivo es para Piaget la esencia de la evolución del conocimiento ontogenético. El principio básico que se encuentra tras el anterior mecanismo es el compromiso con la restauración de la coherencia.

\section{LA CRÍTICA EN LA ENSEÑANZA- APRENDIZAJJE}

En esta sección trataremos de mostrar que la crítica en la enseñanza de las ciencias es un concepto central deI modelo constructivista de enseñanza-aprendizaje.

Comenzaremos por un breve repaso histórico para mostrar cómo la crítica ha sido una preocupación constante de quienes se interesan por cuestiones de enseñanza y aprendizaje.

La estrategia de utilizar contraejemplos como forma de destruir preconceptos se remonta a Sócrates. Según este autor, necesitamos preparar nuestra alma mediante la purificación del intelecto, a fin de permitir el reconocimiento de la verdad evidente al leer en el libro de la naturaleza.

Encontramos en Dewey (1910) uno de los primeros posicionamientos ante la existencia de una clase de 
teorías pedagógicas, que prestan atención a la noción de confusión y conflicto como objetivo instruccional deseado, pues éstas son condiciones necesarias para un cambio cognitivo estable, consistente y coherente. En esta misma línea, Murray (1977), en su trabajo sobre Ia cuestión de la conservación y del papel del conflicto, destaca que este último concepto está relacionado con el proceso de mejora cognitiva. El conflicto como concepto también se apoya en el aumento de la motivación mediante la búsqueda de nuevas informaciones, intentando organizar el conocimiento ya poseído (Berlyne, 1957). Murray (1983) sostiene que el conflicto puede simpiemente no ser percibido por el estudiante, admitiéndolo como una paradoja, un misterio, o percibiéndolo como dos elementos independientes, siendo a veces ignorado uno de estos elementos conflictivos.

Otras investigaciones definen la controversia (Johnson y Johnson, 1979), como las ideas, informaciones, conclusiones, teorías u opiniones de un sujeto, incompatibles con las de otro, al tratar de alcanzar un entendimiento común. Añaden que, al resolver las controversias, los estudiantes necesitan ser capaces tanto de comprender la información de sus oponentes como de entender su perspectiva cognitiva, a fin de organizar e interpretar la información dándole significado. Indican que hay evidencias de que la controversia entre miembros de un grupo y cl conflicto conceptual generado representa un importante factor de crecimiento cognitivo, con una alta calidad en la resolución de problemas y una precisa toma de decisiones o de perspectivas. Con ello se estimulan transiciones a estados más clevados de razonamientos cognitivos y morales, aumenta la creatividad y desencadena a través de las discrepancias un gran cantidad de informaciones y hechos. En definitiva, se favorece el aprendizaje.

Las circunstancias que propician la controversia van desde las preocupaciones debidas a la heterogeneidad de los participantes hasta la facilitación de un clima cooperátivo en la enseñanza, evitándose desenlaces negativos en el sentido de una competitividad destructiva (Johnson y Johnson, 1979; Bainer, 1985). El proceso exige del estudiante el reconocimiento de similitudes, así como de diferencias en las argumentaciones.

En relación con las controversias, Geddis (1991) indaga en mejorar la calidad de los discursos en el aula. Enfatiza que, al tomar una posición defensiva a través del discurso, el sujeto sufre la interferencia de los intereses del protagonista de las discusiones (profesor).

Los estudios de Karmiloff-Smith e Inhelder (1974) con niños de entre 4 y 8 años, y el de Rowell (1983) con adolescentes (high school), ligados a preocupaciones más próximas a una propuesta de enseñanza-aprendizajc atienden al papel del objeto (del experimento) como contraejemplo empírico, juntamente con la influencia de éste en las secuencias de acción y en las transformaciones de las respuestas generadas por los sujetos. Los primeros afirman que la construcción y la generalización de teorías se «muestran dinámicas y de un proceso general»; de ese modo intentan comprender el mecanismo de descubrimiento en acción. Estos estudios concluyen que los sujetos tratan de conservar sus ideas creando teorías independientes para explicar las anomalías, o bien interpretan la refutación como mal uso de la teoría. De forma que ésta no es contestada directamente por los resultados experimentales. Los contraejemplos no se usan como pruebas de las teorías.

Karmiloff-Smith e Inhelder sostienen además que la construcción de falsas teorías o el exceso de generalización (overgeneralization) de éstas es un proceso productivo, pudiendo ser visto como simplificaciones que crean un problema, ignorándose algunos factores confusos. Éstas son sus palabras:

«Es natural del aspecto creativo de los estudiantes, así como de los científicos, la tendencia a explicar Ios fenómenos por medio de una teoría simplificada, la más general y simple posible. No es sorpresa que ambos, científicos y niños, frecuentemente refuten contraejemplos una vez éstos acumulen factores que complican más que simplifican el fenómeno. Los niños procuran mantener el máximo posible sus teorfas iniciales mientras pueden. Incluso, cuando éstas tienen en cuenta los contraejemplos, prefieren crear una teoría independiente de la primera antes de intentar la unificación de todos los eventos envueltos en una única y mayor teoría.»

Extendiendo un poco más esta asociación Feyerabend (1989) dice:

«Los científicos no abandonan teorías en base a evidencias contradictorias; es más, usan teorías falsas todo el tiempo, salvándolas mediante la crítica de los métodos utilizados o mediante hipótesis auxiliares.»

Siegel (1989) propone ligar la racionalidad de la ciencia con el pensamiento crítico y la educación en ciencias. Su objetivo es ante todo defender una visión tradicional de la racionalidad científica vinculada al método científico que busca evidencias. Argumenta que todo objetivo educativo debe supeditarse al pensamiento crítico que evalúa y obra en base a buenas razones, imponiéndolas de manera hábil, garantizando y justificando creencias, proposiciones y acciones. Siegel afirma que el compromiso con la evidencia es básico tanto para la ciencia como para la educación. Así, una educación en ciencias debe fomentar el compromiso con la evidencia e incentivar el pensamiento crítico para conducir al éxito en la iniciación a la ciencia.

Pretendiendo establecer un paralelismo entre Ios proceso epistemológicos y la enseñanza de las ciencias, Silveira (1992) propone relacionar las ideas de Popper sobre el progreso de la ciencia con el proceso de cambio conceptual. Así, el autor presenta una estrategia de enseñanza que trata de sustitir las concepciones alternativas de los estudiantes por las científicas. Su propuesta comienza por explicitar en detalle las predicciones de Ios alumnos en cuanto al empleo de concepciones alternativas que pueden ser corroboradas por la experiencia. Sigue la crítica de dichas concepciones mostrando que determinadas previsiones contradicen la realidad; hay 
también consideraciones sobre las inconsistencias logicas. Al presentar la teoría científica señalando sus antagonismos con las concepciones alternativas, el profesor debe resaltar la capacidad predictiva de la teoría científica para explicar los hechos citados por las concepciones alternativas y que también explica los hechos que entran en conflicto con éstas. Demuestra que la teoría científica permite un mayor alcance y dominio de la realidad que el logrado por las concepciones alternativas.

Para finalizar, Ias investigaciones de Mariani (1987) y Laburu (1993) tratan de asociar el modelo de cambio conceptual con las ideas piagetianas de perturbacióncompensación aplicándolas a contenidos de física de segundo grado. Mariani analiza la evolución de las concepciones espontáneas de alumnos de segundo grado sobre colisiones. Su trabajo integra los resultados procedentes de investigaciones sobre concepciones espontáneas y los obtenidos por Piaget y colaboradores. Laburu (1993) supone que un cambio de compensaciones equivale fundamentalmente a un cambio conceptual. Emplea una lectura de la estructura piagetiana de perturbaciones-compensaciones para describir el conocimiento desarrollado en una situación real de aula. Establecida Ia estructura de compensaciones, el trabajo hace un análisis utilizando las componentes de inteligibilidad, platsibilidad y fructificación de Posner y otros (1982) para entender lo que lleva a aquella lectura de Ias compensaciones piagetianas a estabilizarse en cuanto a la elaboración del conocimiento de los alumnos.

Comenzaremos a detallar los fundamentos generales del modelo constructivista de enseñanza-aprendizaje. Como veremos, dicho modelo atribuye real importancia a la enseñanza crítica.

El principio orientador constructivista supone que el conocimiento no es simplemente transmitido o revelado, sino construido por el propio sujeto a partir de impresiones o nociones que evolucionan de acuerdo con las posibilidades y oportunidades de dicho sujeto y de sus relaciones con otros. Según Driver (1989), el aprendizaje ocurre a través de la implicación activa del aprendiz en la construcción del conocimiento; él es el arquitecto de su propio conocimiento.

En la perspectiva constructivista, los aprendices construyen representaciones mentales del mundo que les rodea, que son usadas para interpretar nuevas situaciones y guiar sus acciones. La consecuencia de dicho pensamiento es que los sujetos (alumnos) parten de conocimientos preestablecidos y cuando observan o interpretan to real lo hacen cargados de esos preconceptos que son muchas veces divergentes del conocimiento científico que se quiere promover (Viennot, 1979; Watts, 1983; Driver, 1989).

Lo que se nota de dicha escuela, que le imprime carácter de conductora de un movimiento, es su postura frente al proceso de enseñanza-aprendizaje. Esta postura determina el establecimiento de un cambio conceptual. Dicho de otra manera, el aprendiz parte frente a un nuevo contenido de conocimientos ya construidos por sí mis" mo, observando dicho contenido a la luz de sus «teorías». Entonces se debe tratar de transformar dicha concepción previa en aquélla que se quiere enseñar.

A fin de favorecer el cambio conceptual, el modelo constructivista ofrece dos estrategias principales (Driver et al., 1992): la primera se funda en la perspectiva del conflicto cognitivo $-y$ puede ser vista como derivada de una visión piagetiana de aprendizaje en la que el aprendiz reorganiza activamente su conocimiento-; la segunda se esfuerza en construir los nuevos dominios de conocimiento a partir de las concepciones de los aprendices, utilizando analogías y metáforas (Clement et al., 1987; Stavy, 1991).

Como el interés de este trabajo se dirige a la primera estrategia, mostremos cómo el conflicto cognitivo, que emplea específicamente la «insatisfacción» (en el sentido de promover una contradicción entre una concepción previa y la evidencia que la contradiga), es el elemento central de esta estrategia.

Posner y otros (1982) establecen que el cambio conceptual está condicionado por cuatro situaciones o condiciones (que la nueva concepción sea satisfactoria, inteligible, plausibble y fructífera) que deben ser satisfechas para que un nuevo y mejor concepto suplante a otro menos apropiado. Ligada a estas condiciones está la componente de ecología conceptual, que suministra el contexto en el cual ocurre el cambio conceptual. Ia naturaleza de esta ecología conceptual indica que la comprensión del individuo es el resultado de una base conceptual coherente, lastrada de elementos como anomalía, analogía y metáforas, compromisos epistemológicos, conceptos y creencias metafísicas y conocimicnto de otros tipos.

Estos cinco elementos impregnan las cuatro condiciones anteriores, formando un «medio ambiente intelectua! activo, semejante a un nicho ecológico. Ambiente en el cual las personas viven (incluyendo creencias culturales, lenguaje, teorias admitidas, hechos y eventos), favoreciendo ciertos conceptos e inhibiendo otros» (Hewson, 1985).

Mientras que, para Posner y otros (1982), las condiciones anteriores forman los cimientos del desarrollo efectivo de un concepto, Hewson (1985) y Hewson-Thorley (1989) entienden como prioritario el cambio conceptual, la toma de consciencia por el aprendiz del estatus ostentado por las anteriores condiciones. De esta forma, el control de dicho cambio de estatus es percibido por el estudiante a través de sentimientos, opiniones, actitudes y reflextones frente a sus concepciones. Strike y Posner (1993), corrigiendo algunas de sus posiciones, apuntan como fundamental la integración de los conceptos con las concepciones articuladas por la ecología conceptual del sujeto; las primeras son parte de las segundas. Aclaran que la estrategia de insertar las concepciones alternativas del estudiante en un «mar de anomalías» a veces no promueve un cambio conceptual, pues al observar el mundo nos inclinamos a verlo de manera consistente con 
nuestras concepciones. Además, recordemos la afirmación de los mismos autores al establecer qué factores no exclusivamente racionales, como los institucionales, los motivacionales, Ios de la visión de la naturaleza y de los valores relacionados con una cuestión dada, que forman parte de la ecología conceptual, deben ser valorados al considerar las dificultades para un cambio conceptual.

Désautels y Larochelle (1990) sostienen que tuna perturbación epistemológica debe dirigir el cambio conceptual. El objetivo de la perturbación es cuestionar el estatus epistemológico de nuestros conocimientos habituales y reconocer el valor de los postulados y reglas científicos. Afirman que los estudiantes, cuando fabrican conceptos, no solamente los crean en base a conocimientos previos ya elaborados, sino también influenciados por una estructura epistémica implícita o inconsciente, que guía la actividad cognitiva. Tal estructura epistémicaestá constituida por postulados epistemológicos, creencias metafísicas, reglas metodológicas, hábitos de pensamiento y está incorporada en el lenguaje del día a día, siendo espontáneamente empleada en la construcción de explicaciones del mundo natural. Lo necesario es cuestionar (perturbación epistemológica) estos postulados, creencias, reglas, etc., a fin de que los individuos no reinterpreten conocimientos curriculares bajo el contexto de sus estructuras epistémicas, atribuyendo significados a los conocimientos científicos diferentes de aquéllos que se desea oficializar.

Las afirmaciones anteriores establecen Ios fundamentos del modelo constructivista e indican que un cambio conceptual pasa por un cambio de estatus no sólo de las cuatro componentes mencionadas sino también de una substitución, reorganización, jerarquización e innovación de elementos de la ecología conceptual que estén integrados a los conceptos que se quiere inhibir por un lado y favorecer por otro.

Con respecto al conflicto que destacamos, la condición que fija la insatisfaccion frente a un concepto previo se convierte en uno de los elementos imprescindibles para el cambio conceptual. Esta condición está relacionada con las expectativas insatisfechas, esto es, con las contradicciones entre enunciados de observación y enunciados teóricos. Después de esto, la relación de coherencia entre los conceptos y su ecología conceptual es de fundamental importancia en dicho proceso de cambio conceptual.

Los trabajos de Rowell y Dawson (1985) y de Hewson (1990), preocupados por la enseñanza-aprendizaje, sugieren estrategias divergentes para emplear aquel concepto. Mientras que el segundo busca el conflicto inmediatamente después de hacer explícitas las concepciones alternativas, el primero se inspira en una perspectiva lakatosiana. En ésta, Lakatos establece la condición para que un problema sea considerado un contraejemplo. Esta condición seré satisfecha en la medida en que un «programa de investigacións" posee una teoría que resuelve el problema no solucionado por la(s) teoría(s) del «programa de investigación» en cuestión. En posesión del contraejemplo, el «programa de investigación» muestra su éxito en relación con un programa rival (que no consigue solucionar el problema) cuando emplea el contraejemplo como refutador (visto retrospectivamente) de éste último (Lakatos, 1979). Para Lakatos, el contraejemplo solamente se establece a partir de la comparación entre "programas de investigación» que logran la solución del problema y aquéllos que no consiguen afrontarlo. En caso de que no exista por lo menos un "programa de investigación» con éxito, el contraejemplo deja de ser considerado refutación para los programas que no lo solucionen satisfactoriamente. A partir de aquí Rowell y Dawson (1985) establecen la importancia del conflicto cognitivo como estrategia de enseñanza-aprendizaje solamente cuando el alumno posea una teoría mejor frente a sus concepciones alternativas. Hewson (1985) también mantiene que, para la efectividad de un conflicto cognitivo durante la instrucción, es imprescindible el compromiso de los alumnos con la consistencia interna y la generalidad. Las decisiones del estudiante cuando comprometen la consistencia interna lo llevarán a una situación de conflicto. Por otro lado, su compromiso con la generalización hará que el conflicto lo conduzca al rechazo de una concepción alternativa volviéndola, en las componentes para el cambio conceptual de Posner y otros (1982), menos plausible.

Es posible, en suma, verificar que una consecuencia deI programa constructivista es la valoración del pensamiento crítico. La coherencia y la generalidad de las afirmaciones son condiciones buscadas a partir de la confrontación directa de las ideas del aprendiz con la contradicción.

\section{CONCLUSIONES}

Este trabajo ha tratado de mostrar que la coherencia es un invariante epistémico ansiado respecto a la evolución fillogenética y ontogenética.

Se ha hecho ver que las contradicciones en la ciencia, consideradas como tales, deben ser superadas y que esto está íntimamente ligado al aumento del conocimiento. En lo que se refiere al sujeto, hemos visto que la contradicción es también un factor relevante del desarrollo cognitivo.

En lo tocante a las acciones pedagógicas, se ha sugerido que el desarrolio del pensamiento crítico es un parámetro que debe ser considerado en el aula. Ha quedado implícito que, para alcanzar el objetivo pretendido, es fundamental una actitud crítica -que valore la comparación entre una prevision o expectativa y el dato observable que a ésta se contraponga - para que ese pensamiento pueda ser explorado, un procedimiento que puede alcanzar el profesor cuando imponga situaciones perturbadoras (problemas) a sus alumnos ${ }^{3}$. Aquí debe estar presente la exigencia o el compromiso del aprendiz con la coherencia, con la consistencia interna de los conceptos y con la generalidad de los pensamientos. 
De la misma forma que en la epistemología, las perspectivas piagetiana y constructivista indican que una contradicción no ileva obligatoriamente a la superación de una concepción. El aprendiz se protege de la contradicción de varias maneras: compensación alfa piagetiana o estatus más elevado de una concepción alternativa frente a la científica que se pretende promover. EI modelo constructivista camina en el sentido de que un restultado satisfactorio para el cambio conceptual será mayor cuanto mayor sea la habilidad del profesor para debilitar la coherencia de las concepciones alternativas de los estudiantes, resaltando la limitación y la inconsistencia de éstas frente al alcance de las científicas. Ello obliga a reconsiderar los «artefactos cognitivos» de la ecología

\section{NOTAS}

' Para I audan son considerados problemas tanto los conceptuales como los empíricos.

2. Un programa de investigación consiste en unas reglas metodológicas, cuyo objetivo es informar de los caminos que deben ser evitados (heurística negativa) -para no alterar los fundamentos del programa de investigación-o de los caminos que deben ser recorridos -modificando el programa.

${ }^{3}$ Problemas entendidos aquí como contradicciones. Creemos que toda contradiccion genera un problema. Sin embargo, ni todo problema puede ser visto como una contradicción, pues

\section{REFERENCIAS BIBIIOGRÁFICAS}

BAINER, D.L. (1985). What to do when people disagree: addressing ideational pluralism in science classes. Sci. Educ., Vol. $69(2)$, pp. $171-183$

BERLYNF, D., (1957). Uncertainty and conflict: a point of contact between information-theory and behavior-theory concepts. Psychological Review, 64, pp. 329-339.

CARVAI,HO, A.M.P., CASTRO, R.S., LABURU, C.E. y MORTIMER, E.F. (1993). Pressupostos epistemológicos para a pesquisa em ensino de ciências. Cadernos de Pesquisa, 82, pp. $85-89$.

CASTRO, A.D. (1974). Piaget e a didáctica de ensaios. Sao Paulo: Ediçăo Saraiva.

CLEMENT et al. (1987). Overcoming students' misconceptions in physics: the role of anchoring intuitions and analogical validity. Proceedings of the Second International Seminar. Misconceptions and Educational Strategies in Science and Mathematics, 3, pp. 84-97.

DESAUTELS, J.y LAROCHELI.E, M. (1990). A constructivist pedagogical strategy: the epistemological disturbance (experiment and preliminary results), en More History and Philosophy of Science in Science Teaching. Proceedings of the First International Conference. Edited by Dom Emil Herget, pp. 236-257. Florida State University. conceptual de las concepciones alternativas, mostrar sus contradicciones y buscar el fortalecimiento de los conceptos científicos, junto con la ecología conceptual que los impregna.

Al tratar de promover contradicciones conceptuales y empíricas $y$, en un proceso dialéctico, hacer que sus aprendices rescaten constantemente la coherenciá conceptual, el profesor estará astumiendo una enscñanza crítica y promocionando sujetos más críticos.

Este trabajo ha tratado de realzar la importancia del profesor en su compromiso con el pensamiento critico y ha intentado indicar cómo podría ser llevado a cabo en el aula.

muchas veces un problema a resolver no proviene de una previsión insatisfecha - empíricamente hablando- o de la de conciencia de una inconsistencia conceptual. Como ejemplo. sea el siguiente problema: ¿Cómo debo hacer para atravesar este río? Resolver este problema no significa necesariamente que estén presentes las afirmaciones anteriores.

* Este artículo ha sido traducido del portugués por Víctor $M$. Álvarez.

DEWEY, J. (1910). How to think. Boston: Heath.

DRIVER, R. (1989). Students' conceptions and the learning of science. Int. J. Sci. Educ, Vol. II(S), pp. 481-490.

DRIVER ct al. (1992). Teaching for conceptual change: a review of strategies, en Duit, R. et al. (eds.)., Research in Physics Learning: Theoretical Issues and Empirical Studies. Proceedings of an International Workshop Held at the University of Bremen, March 4-8, 199, pp. 310-329.

DYKSTRA, D.I.(1992). Studying conceptual change: constructing new understandings, en Duit, R. et al. (eds.)., Research in Physics Learning: Theoretical lssues and Empinical Studies. Proceedings of an International Workshop Held at the University of Bremen, March 4-8, 1991, pp. 40-58.

FEYERABEND, P. (I989). Contra o método. Rio de Janeiro: Ed. Francisco Alves.

FRANCO, C. y COIINVAUX-DE-DOMINGUHZ, D. (1992) Genetic epistemology, history of science and science education. Science \& Education, 1, pp. 255-271.

GEDDIS, A.N.(1991). Improving the quality of science classroom discourse on controversial isstes. Sci. Educ., 75(2), pp. 169-183. 
GIL, D. (1993). Contribución de la historia y de la filosofía de las ciencias al deasrollo de un modelo deenseñanza-apendizaje como investigación. Enseñanza de las Ciencias, Vol. 11(2), p). 197-212.

GIL, D. y CARRASCOSA, I. (1985). Science learning as a conceptual and methodological change. Eur. J. Sci. Educ., Vol. $7(3)$, pp. 23l-236.

GII., D., DUMAS-CARRÉ, A. et al. (1990). Paper and pencil problem solving in the physical sciences as an activity of research. Studies in Science Education, 18, pp. 137-151.

HEWSON, P. W. (1985). Epistemological commitments in the learning of science: examples from dynamics. Eur. J. Sci. Educ., Vol. 2(2), pp. 163-172.

HEWSON, P.W. (1990). La enseñanza de «fuerza y movimiento como cambio conceptual». Enseñanza de las Ciencias, 8(2), pp. $157-171$.

HEWSON, P.W. y HEWSON, M.G.A.B. (1992). The status of students' conceptions, en Proceedings of an International Workshop Held at University of Bremen. Research in Physics Learning, pp. 59-73.

HEWSON, P.W. y THORLEY, N.R. (1989). The conditions of conceptual change in the classroom. Int. J. Sci. Educ., Vol. 1, pp. $541-553$.

INHEIDER, B, GARCÍA, R. y VONECHE, I. (1978). Epistemologia genética e equlibração. Lisboa: Horizonte Universitário

JOHNSON, D. y JOHNSON, R.T. (1979). Conflict in the classroom: controversy and learning. Review of Educ. Research, Vol. 49(1), pp. 51-70.

KARMILOFF-SMITH, A. E INHELDER, B. (1974). If you want to get ahead, get a theory. Cognition, 3, pp. 195-212.

KUHN, T.S.(1987). A estrutura das revoluçöes científicas. Sao Paulo: Editora Perspectiva

I.ABURU, C.E. (1993). A construção do conhecimento em sala de aula. Tesis de Doctorado/Sao Paulo. FEUSP.

I.AKATOS, I. y MUSGRAVE, A. (1979). A crítica e o desenvolvimento do conhecimento. Sao Paulo: Editora Cultrix.

I.AUDAN, L. (1977). Progress and its problems, toward a theory of scientific growth. Berkeley y Los Angeles: Unjv. of California Press, Ltd.

MARIANI, M.C. (1987). A evolução das concepçōes espontâneas sobre colisóes. Dissertação de Mestrado/Sao Paulo. IFUSP/ FEUSP.

MURRAY, F.B. (1983). Equilibration as cognitive conflict. Developmental Review, Vol. 3, pp. 54-61.

PIAGET, J. (1970). A constrtıção do real na criança. Río de Janeiro: Zahar.

PIAGET, J. (1974). Investigaciones sobre la contradicción. Madrid: Siglo Veintiuno Editores.
PIAGET, J. (1977). O desenvolvimento do pensamento equilibração das estruturas cognitivas. Lisboa: Publicaçōes Dom Quixote.

PIAGET, J. (1980). Lógica e conhecimento científico, en Enciclopédia de la Pleiade, pp. 17-114, VI. Portugal: Livraria Civili-zação Editora.

PIAGET, J. (1983). Sabedoria e ilusões da filosofia, en Pensudores. pp. 7]-208. Sao Paulo: Victor Civita.

PIAGET, J. y GARCÍA, R. (1984). Psicogénesis e historia de la ciencia. México: Siglo Veintiuno Editores.

POPPER, K.R. (1972). A lógica da pesquisa cientifica. Såo Paulo: Cultrix.

POPPER, K.R. (1972a). Conjecturas e refutaçöes. Brasilia: Editora Universidade de Brasília.

POPPER, K.R. (1987). O realismo e o objectivo da ciência. Lisboa: Publicaçöes Dom Quixote,

POSNER, G.J., STRIKE, K.A., HEWSON, P.W. y GERTZOG, W.A. (1982). Accommodation of scientific conception: Toward a theory of conceptual change. Sci. Educ., 66(2), pp. $221-227$.

ROWELL, I.A. (1983). Laboratory counter examples and the growth of understanding in science. Eur. J. Sci. Educ., 5(2), pp. 203-215.

ROWELL, J.A. (1983a). Equlibration: developing the beart core of the piagetian research program. Hum. Dev, 26, pp. 61-71.

ROWELL, J.A. (1989). Piagetian epistemology: equitibration and the teaching of science. Synthese, 80, pp. 41-162.

ROWELL, I.A. y DAWSON, L. (1985). Equilibration, conflict and instruction: a new class-oriented perspective. Eur. $J$. Sci. Educ., 7(4), pp. 331-344.

SIEGEL, R. (1989). The rationality of science, critical thinking, and science education. Synthese, 80, pp. 9-41.

SłLVEIRA, F.L. (1992). A filosofía da ciência e o ensino de ciências, en Aberto. Brasilia, año 11 , N 55: 36-41.

STRIKE, K.A. y POSNER, G.J. (1993). A revisionist theory of conceptual change, en Duschi, R. y Hamilton, R. (eds.), Philosophy of Science and Educational theory and Practice. Albany, NY: State Uni. of NY Press.

VIENNOT, L. (1979). Spontaneous reasoning in elementary dynamics. Eur. J. Sci. Educ., 1, pp. 205-221. Londres y Filadelfia.

VUIK, R. (1981). Overview and critique of Pinget's genetic epistemology 1965-1980, 1, 264 p. Londres: Academic Press.

WATTS, D.M. y ZYLBERSTAIN, A. (1981). A survey of some children's ideas about force. Phys. Educ., 16, pp. 360365 .

WHITE, R.T. y GUNSTONE, R.F. (1989). Metalearning and conceptual change. Int. J. Sci. Educ., 11, pp. 577-586. 
